\title{
A MICROFABRICATION-BASED APPROACH TO QUANTITATIVE ISOTHERMAL TITRATION CALORIMETRY
}

\author{
Bin Wang ${ }^{\dagger}$ Y Yuan Jia and Qiao Lin* \\ Department of Mechanical Engineering, Columbia University, New York, NY
}

\begin{abstract}
Isothermal titration calorimetry (ITC) directly measures heat evolved in a chemical reaction to determine equilibrium binding properties of biomolecular systems. Conventional ITC instruments are expensive, use complicated design and construction, and require long analysis times. Microfabricated calorimetric devices are promising, although they have yet to allow accurate, quantitative ITC measurements of biochemical reactions. This paper presents a microfabricationbased approach to integrated, quantitative ITC characterization of biomolecular interactions. The approach integrates microfabricated differential calorimetric sensors with microfluidic titration. Biomolecules and reagents are introduced at each of a series of molar ratios, mixed, and allowed to react. The reaction thermal power is differentially measured, and used to determine the thermodynamic profile of the biomolecular interactions. Implemented in a microdevice featuring thermally isolated, well-defined reaction volumes with minimized fluid evaporation as well as highly sensitive thermoelectric sensing, the approach enables accurate and quantitative ITC measurements of protein-ligand interactions under different isothermal conditions. Using the approach, we demonstrate ITC characterization of the binding of 18-Crown-6 with barium chloride, and the binding of ribonuclease A with cytidine 2'-monophosphate within reaction

\footnotetext{
* To whom correspondence should be addressed. Phone: 1-212-854-1906; E-mail: qlin@ columbia.edu.

${ }^{\dagger}$ Presently with BD Medical, Sandy, UT.
} 
volumes of approximately $0.7 \mu \mathrm{L}$ and at concentrations down to $2 \mathrm{mM}$. For each binding system, the ITC measurements were completed with considerably reduced analysis times and material consumption, and yielded a complete thermodynamic profile of the molecular interaction in agreement with published data. This demonstrates the potential usefulness of our approach for biomolecular characterization in biomedical applications.

Keywords: Biomolecular interaction, binding isotherm, differential calorimetry, isothermal titration calorimetry, microfabrication 


\section{INTRODUCTION}

Isothermal titration calorimetry (ITC) is a differential calorimetric method that directly measures heat evolved in a chemical reaction as a function of molar ratio of reactant concentrations. A solution-phase, label-free method, ITC allows determination of all equilibrium binding parameters with a single set of experiments for characterization of biomolecular interactions [1] and is widely used in basic biochemical studies [2-4], as well as practical applications such as drug discovery and biotherapeutics development [5-7].

Conventional methods for differential calorimetry, including ITC, while well established and effective, are expensive, use complicated design and construction, and require long analysis times [8]. Microfabrication technology holds the potential to drastically improve calorimetric measurements [9]. Existing microfabricated calorimetric devices (i.e., calorimetric microdevices), however, are often limited to solid- or gas-phase samples [10,11]. Calorimetric microdevices for liquid-phase samples [12-15] have combined microfluidic handling of liquid samples with microfabrication-based thermal transduction, for example via infrared (IR) thermography [16], mechanical resonance [17], or thermal sensing using integrated thin-film resistors [18] or thermopiles [19-24]. While some of these devices have employed differential measurements of the reaction heat [21-24], most are based on more straightforward but less accurate nondifferential configurations [9-20]. In efforts with relevance to ITC, heat fluxes evolved in the chemical reaction were measured by continuous in-channel delivery of reactants at varying flow rates, and used to calculate the enthalpy change [25]. By sequential injection of small droplets of a reactant into a larger droplet of the other reactant, the reaction heat per injection was also measured in real time [26, 27]. Unfortunately, because of inadequate control of the reaction conditions (e.g., temperature control and reagent titration), these devices were limited to 
measuring acid-base reactions at room temperature and did not allow determination of binding isotherms as required for ITC studies. In an attempt to enable ITC measurements, the reaction heat from droplets of reactants at varying concentrations was measured to obtain the binding isotherm [28]. This allowed the determination of the appropriate binding parameters; however, the measurement accuracy could be affected by evaporation of the droplets, which can induce significant thermal disturbances as well as losses of the reactant solution volume. Indeed, accurate, quantitative ITC measurements of biochemical reactions, in particular protein-ligand interactions under different isothermal conditions, have not yet been demonstrated in welldefined volumes of microdevices.

This paper presents, for the first time, a microfabrication-based approach to integrated, quantitative ITC characterization of biomolecular interactions. The approach integrates microfabricated differential calorimetric sensors with microfluidic titration, in which microliterlevel reactants are mixed at a series of molar ratios in a constant volume and at a controlled temperature, while the reaction heat is measured. A microdevice is used to implement the approach and consists of thermally isolated microchambers with minimized liquid evaporation to provide a constant-volume reaction environment, passive micromixers for titration of reactants, and a thermoelectric sensor for differential detection of the reaction heat. Quantitative ITC measurements of biomolecular interactions can thus be performed in the integrated device at user-specified temperature set points, within considerably reduced analysis times, without dilution-induced baseline shifts that often affect conventional ITC instruments, and potentially at low cost. Measurement data are then used to quantitatively determine a complete thermodynamic profile of biomolecular binding, including the equilibrium binding constant, stoichiometry and molar enthalpy change. This is demonstrated with the binding of 18-Crown-6 (18-C-6) with 
barium chloride $\left(\mathrm{BaCl}_{2}\right.$ and the binding of ribonuclease $\mathrm{A}$ (RNase $\mathrm{A}$ ) with cytidine 2'monophosphate (2'CMP), in $\sim 0.7 \mu \mathrm{L}$ reaction volumes and at concentrations down to $2 \mathrm{mM}$, with ITC measurements completed within 30 min compared to $1 \sim 2$ hours for conventional instruments, Thermodynamic binding properties are obtained for each system and are found to agree with published data.

\section{EXPERIMENTAL SECTION}

\subsection{Microfabrication-Based ITC Approach}

Consider a solution-based biochemical binding system in which, for example, a receptor $(M)$ binds with ligand $(X)$ in equilibrium:

$$
\alpha M+\beta X \rightleftarrows M_{\alpha} X_{\beta}
$$

where $\alpha$ and $\beta$ are the stoichiometric coefficients of the receptor and the ligand, respectively, and $M_{\alpha} X_{\beta}$ is the complex resulting from the binding event. In ITC, the ligand is titrated, i.e., successively added in known aliquots, into the receptor, while the heat evolved during the reaction is measured as a function of the ratio of molar concentrations of the reactants. This information is then used to calculate the thermodynamic properties of the reaction. These include the equilibrium binding constant $K_{B}=C / M^{\alpha} X^{\beta}$ where $M$ and $X$ also respectively denote the equilibrium concentrations of the receptor and ligand, and $C$ that of the complex $M_{\alpha} X_{\beta}$, as well as the stoichiometry $(N=\beta / \alpha)$ and molar enthalpy change of binding $(\Delta H)[29,30]$. The reaction heat in the binding event can be expressed as [31]: 


$$
Q=\frac{N M_{T} \Delta H V_{0}}{2}\left[1+\frac{r}{N}+\frac{1}{N K_{B} M_{T}}-\sqrt{\left(1+\frac{r}{N}+\frac{1}{N K_{B} M_{T}}\right)^{2}-\frac{4 r}{N}}\right]
$$

which is a function of the ligand-receptor molar ratio $r=X_{T} / M \mathrm{~T}$ where $M_{t}$ is the total concentration of the receptor (both free and bound)) and $X_{t}$ the total concentration of the titrated ligand, in the reaction volume $\left(V_{0}\right)$.

The reaction heat is determined using a differential measurement scheme, where two identically sized microchambers (below) are used for measuring heat in the presence (ligandreceptor system) and absence of the ligand (reference system), respectively. Both chambers are initially filled with a solution of the receptor, while the reaction and reference chambers are respectively titrated with a ligand and pure buffer. The reaction, either exothermic or endothermic, induces a thermal power difference between the calorimetric chambers: $\Delta P=P_{s}-P_{r}$, where $P_{s}$ and $P_{r}$ are the thermal power in the reaction and reference chambers, respectively. A thermoelectric sensor can thus detect the differential thermal power, or the difference in the thermal power in the chambers [32]

$$
\Delta P=\frac{\Delta U}{S}
$$

where $\Delta U$ is the output from the thermoelectric sensor and $S$ is the device's responsivity, i.e., the output electrical voltage generated by unit differential thermal power [23] .

The ITC microdevice implementing this approach consists of a microfluidic structure bonded to a thermal control and sensing substrate. The microfluidic structure includes two calorimetric chambers fabricated of polydimethylsiloxane (PDMS), which are each situated on a freestanding polyimide diaphragm of the thermal substrate and surrounded by air cavities 
embedded in the sidewalls for effective thermal isolation (Figure 1a). The chambers are also each connected to the associated inlet through a passive micromixer, which uses herringboneshaped ridges in a serpentine channel to generate a chaotic flow pattern that induces the mixing of the incoming liquid streams [33]. On the thermal substrate, the freestanding diaphragms are each integrated with a thin-film antimony-bismuth $(\mathrm{Sb}-\mathrm{Bi})$ thermopile, which produces a voltage between its hot and cold junctions that are respectively located underneath the centers of the reaction and reference chambers for differential power determination (Figure 1(a)). The diaphragms are also each integrated, within the areas occupied by the chambers, with a thin-film resistive micro temperature sensor and a heater, which are used for in-situ monitoring of the chamber temperature, as well as for on-chip device calibration [23].

During ITC measurements, the microdevice temperature is controlled at a desired setpoint (section 2.2). First, ligand-free buffer and receptor solution are introduced into both the reaction and reference chambers through the respective micromixers, and the voltage output from the thermopile is measured to obtain a baseline. With the absence of the ligand, the baseline serves as a control for the overall ITC measurement. Next, the ITC measurement of ligand-receptor binding is performed. Ligand and receptor molecules, which are typically in pure form and dissolved in well-conditioned buffer [34], are introduced at a given molar ratio into the microdevice, first mixed in the appropriate micromixer and then delivered into the reaction chamber. In the meantime, ligand-free buffer and receptor solutions are introduced, mixed, and delivered into the reference chamber. By the differential design of ITC, the thermal power in the reference chamber, which is used as a control for the ligand-receptor binding measurement, is subtracted from the thermal power in the reaction chamber [35]. The resulting differential thermal power between the chambers, which reflects the net contributions of ligand-receptor 
binding reaction, is determined from the thermopile voltage (corrected by subtraction of the baseline) according to Eq. (3). Results from these measurements at a series of ligand-receptor molar ratios are used to compute the binding parameters such as the stoichiometry, enthalpy of the binding, and equilibrium binding constant according to Eq. (2).
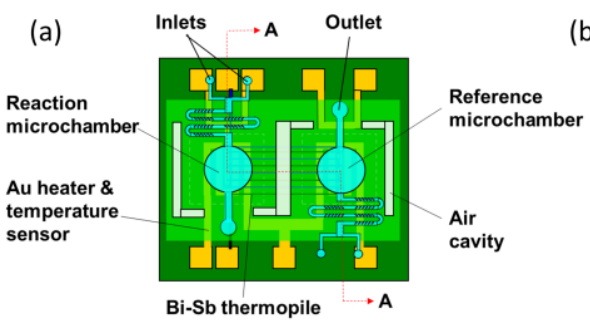

(b)

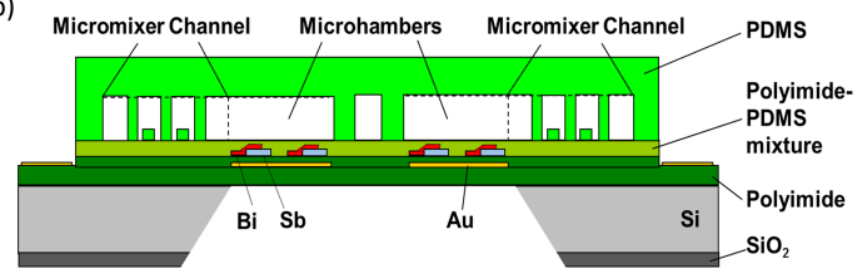

Figure 1. Schematic of the ITC microdevice consisting of a pair of calorimetric chambers respectively holding reactant and reference solutions. The chambers are each situated on a freestanding diaphragm and flanked between air cavities for thermal isolation. The diaphragms are each integrated with a thermopile as well as resistive microheaters and temperature sensors (a) Top view, and (b) cross-sectional view (cross section A-A defined in the top view).

\subsection{Microdevice Fabrication and Testing}

The fabrication of the ITC microdevice followed a process described in detail elsewhere [23]. Briefly, a thermal substrate and a microfluidic structure were fabricated individually and bonded together. The fabricatication of the thermal substrate started with spin-coating and curing a $6 \mu$ m-thick polyimide film on a silicon wafer, which had been thinned down from the backside in the regions corresponding to the calorimetric chambers. Thin films of chromium/gold (7/200 $\mathrm{nm})$ as well as $\mathrm{Sb}$ and $\mathrm{Bi}(0.5$ and $1.2 \mu \mathrm{m})$ were successfully deposited and patterned, and then passivated with thin polyimide layers $(2 \mu \mathrm{m})$, to obtain the gold microheaters and temperature 
sensors as well as the $\mathrm{Sb}-\mathrm{Bi}$ thermopile junctions. In parallel, the calorimetric chambers (diameter: $2 \mathrm{~mm}$; height: $180 \mu \mathrm{m}$ ), chaotic micromixers, and reagent-handling microchannels were fabricated in a poly(dimethylsiloxane) (PDMS) sheet using soft lithography. The microfluidic structure was then bonded to the thermal substrate via an intermediate layer of polyimide-PDMS mixture ( $\sim 2 \mu \mathrm{m}$ thick). Finally, $\mathrm{XeF}_{2}$ gas-phase etching was used to remove the thinned-down silicon below the polyimide, which then formed free-standing diaphragms below the calorimetric chambers. Images of a fabricated microdevice are shown in Figure 2 (Panels a and b).

The test setup for the ITC microdevice primarily consisted of instrumentation for thermoelectric measurements and titration-enabling reagent delivery, as well as a temperaturecontrolled thermal enclosure in which the device was housed (Figure 2(a)). During experiments, the microheaters were driven by a DC power supply (Agilent E3631A) and the temperature sensors were interrogated by a digital multimeter (Agilent 34410A). The thermopile output voltage was measured by a nanovoltmeter (Agilent 34420A). The temperature monitoring and thermoelectric measurement were automated by a personal computer via a LabVIEW-based program.

(a)

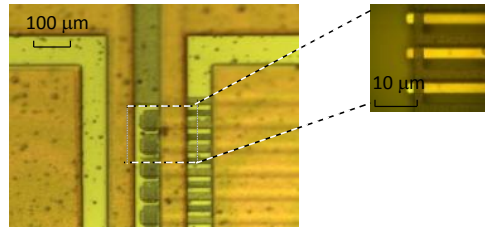

(b)
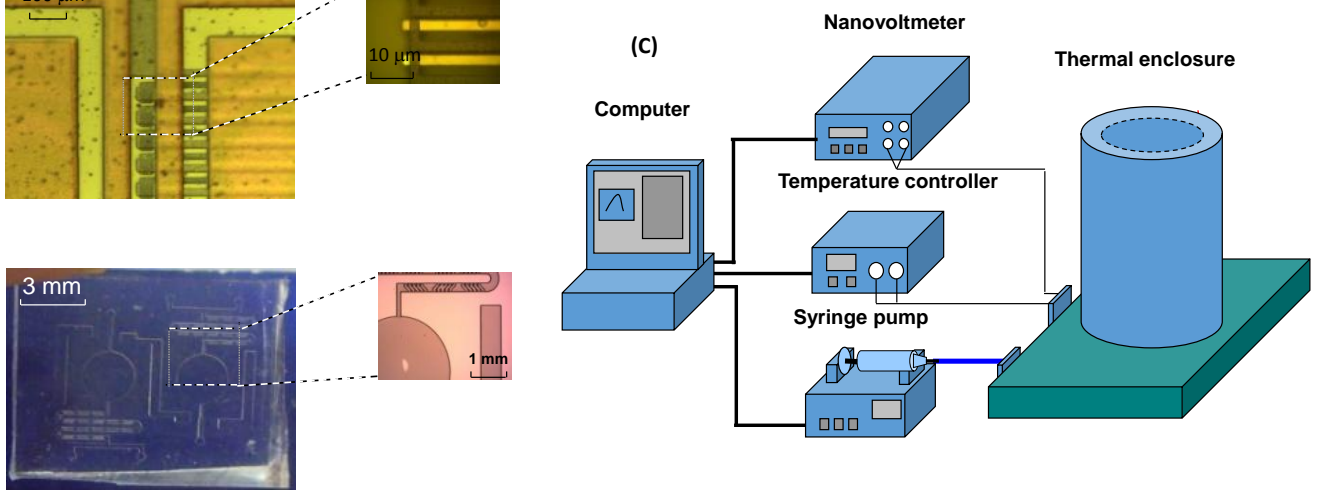
Figure 2. Fabricated ITC microdevice and test setup. (a) Images of the thermopile junctions and temperature sensor. (b) Calorimetric chambers and micromixers. (c) ITC measurement setup.

Solutions of the ligand and receptor, at the desired concentrations, were each preloaded using a syringe pump (KD Scientific, KDS 220) into a Teflon access tubing, and form airseparated segments (each $0.5 \mu \mathrm{L}$ in volume) therein (Figure 3 ). The tubings were then connected to the two inlets for the reaction chamber. From the tubings, the ligand and receptor segments were introduced successively in pairs into and mixed in the appropriate micromixer, and then delivered into the reaction chamber. The ligand-receptor molar ratio was determined from the corresponding ligand and receptor concentrations in the segments. Similarly, two other identical Teflon access tubings were preloaded with segments of receptor and buffer solutions, respectively, and were delivered into the reference chamber. Introduction of ligand, receptor and buffer into the chambers was synchronized using a multi-channel syringe pump (KD Scientific, KDS 220). When appropriate, pure buffer solution and sterile water were also being loaded between the reactant segments to clean the chambers. The positioning of the segments was adjusted as needed after disconnecting the driving syringe and the access tubings.

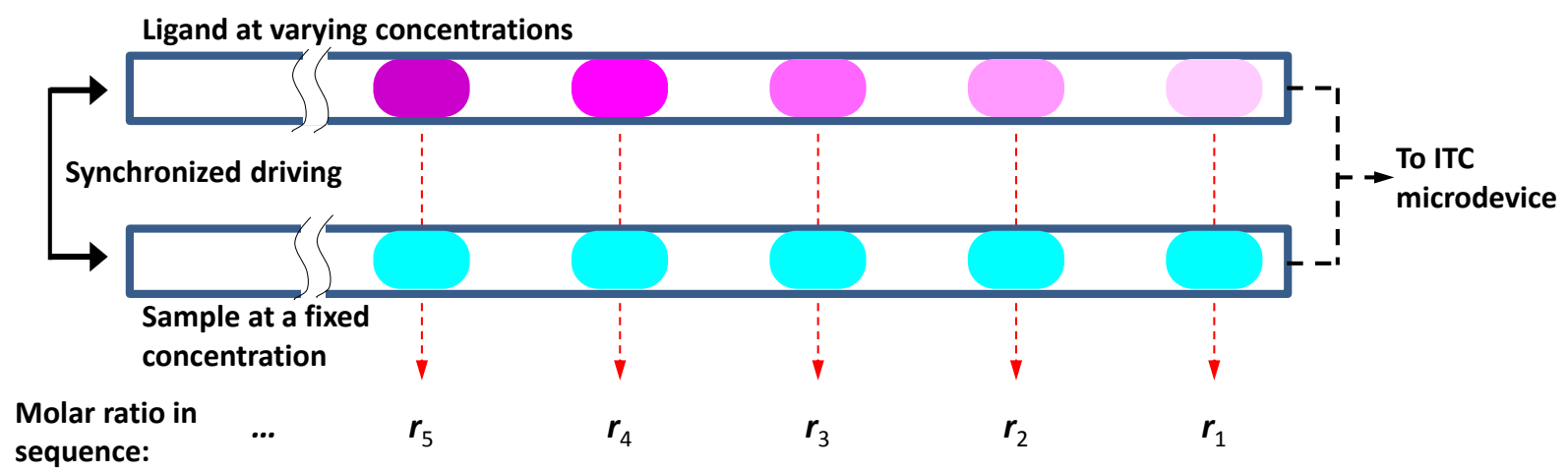


Figure 3. Scheme for varying the molar ratio of the ligand to the receptor: segments of ligand (at differential concentrations) and receptor (at a fixed concentration) were preloaded in access tubes for sequential delivery into the ITC microdevice.

The thermal enclosure was custom-built to house the device (Figure 2(b)), shielding the device from ambient thermal disturbances and providing a low-noise measurement environment with controlled, uniform temperature [23]. Inside the enclosure, the device was placed on a support stage, which, serving as a temperature-controlled platform, was elevated from the base, which in turn was placed on a thick rubber sheet under the base for suppression of mechanical vibration. Multi-port fluidic feedthrough was achieved through the base. The temperature of the device stage (and hence the temperature of the microdevice) was controlled via a Peltier heater (Marlow Industries, XLT2418), a resistive temperature detector (Omega Engineering, F2020) attached to the device stage, and a commercial temperature controller (Lakeshore Model 331).

To investigate the microdevice's steady-state and transient responses to a differential thermal power, the integrated microheaters were used to generate a known, constant thermal power in the reaction chamber, and the response from the thermopile output was recorded [23]. The transient device response to a step thermal power allowed determination of the device's thermal time constant. The relationship between the steady-state thermopile voltage and the applied differential thermal power was used to determine the device's responsivity, thereby allowing its calibration. The microdevice was then used for ITC measurements. During the device characterization and ITC measurements, the on-chip micro-temperature sensors were used to monitor the temperatures of the calorimetric chambers. The ligand, receptor, and buffer 
solutions used in all measurements were degassed with a vacuum chamber built in-house and metered by micropipettes (Eppendorf) before they were loaded in the access tubes.

Materials. For microdevice fabrication LOR resists and SU-8 (2000, 2025, and 2075 series) negative photoresist was purchased from Microchem Corp. (Westborough, MA). Polydimethylsiloxane (PDMS) was obtained from Robert McKeown Company (Somerville, NJ). Polyimide (PI-2610 and PI-2611series) was purchased from HD Microsystem (Parlin, NJ). Antimony and bismuth (99.999\% in purity) were purchased from Kurt J. Lesker Company (Pittsburgh, PA), and silicon wafers were purchased from Silicon Quest International, Inc. (San Jose, CA). For testing the ITC microdevice, 18-Crown-6 (18-C-6, molecular weight: 264.32 Da, $\geq 99.0 \%$ in purity) and barium chloride $\left(\mathrm{BaCl}_{2}\right.$, molecular weight: $208.23 \mathrm{Da}, \geq 99.999 \%$ in purity) were purchased from Sigma (St. Louis, MO), and were diluted with cell culture grade distilled water (Thermo Fisher Scientific, Grand Island, NY) to obtain desired concentrations of 18-C-6 (5 mM) and $\mathrm{BaCl}_{2}(0.5-10 \mathrm{mM})$. Ribonuclease A (RNase A, $\left.13.7 \mathrm{kDa}\right)$ and Cytidine-

2'- monophosphate (2' CMP, 323.20) were obtained from Prof. Jonathan B. Chaires (University of Louisville, USA) as a gift, and prepared at desired concentrations ( $2 \mathrm{mM}$ for RNase A, and 0.2-4 mM for 2'CMP).

\section{RESULTS AND DISCUSSION}

The ITC microdevice used in the experiments had a 50-junction Sb-Bi thermopile and two calorimetric microchambers with a center-to-center separation of $4 \mathrm{~mm}$. Each chamber had a cylindrical shape with a height of $150 \mu \mathrm{m}$ and a diameter of $2.5 \mathrm{~mm}$, and thus a volume of approximately $0.7 \mu \mathrm{L}$. The passive chaotic micromixers each had a serpentine microchannel 
(width: $200 \mu \mathrm{m}$, height: $150 \mu \mathrm{m}$, total length: $15 \mathrm{~mm}$ ) with herringbone-shaped ridges on the ceiling. The ridges each had a width of $40 \mu \mathrm{m}$, a height of $50 \mu \mathrm{m}$, an orientation angle of $60^{\circ}$ with respect to the channel sidewall, and an edge-to-edge distance between adjacent ridges of 30 $\mu \mathrm{m}$. These micromixer dimensions were chosen based on an experimentally verified model [33], to allow the ligand and receptor solutions introduced into each micromixer to be mixed with sufficient $(\geq 90 \%)$ concentration uniformity at the mixer exit. The nominal resistances of the integrated resistive microheaters and temperature sensors were $40 \Omega$ and $55 \Omega$, respectively.

\subsection{Characterization of Thermal Responses}

We first investigated the response of the ITC microdevice to the application of a constant differential power of $90 \mu \mathrm{W}$, and its removal once the device output voltage reached a steady value. The measured device output (i.e., thermopile voltage) from the application and removal of the differential power, was fitted to first-order exponential increase and decay functions, respectively. The thermal time constants for the temperature rise and decay processes were determined to have approximately the same value of $1.5 \mathrm{~s}$ (Figure $4 \mathrm{a}$ ), about one order-ofmagnitude smaller than those of conventional instruments. In addition, the device's steady-state response was found to depend on the differential power in a highly linear manner, yielding an approximately constant responsivity of $S=4.9 \mathrm{mV} / \mathrm{mW}$ (Figure $4 \mathrm{~b}$ ). We also obtained the device's responsivity at different controlled ambient temperatures provided by the thermal enclosure from 20 to $45^{\circ} \mathrm{C}$, and found that it remained constant with a relative standard deviation of less than $3 \%$. Thus, $S=4.9 \mathrm{mV} / \mathrm{mW}$ was subsequently used as a calibration constant in all 
ITC measurements to determine the differential thermal power between the calorimetric chambers from the measured thermopile voltage.

(a)

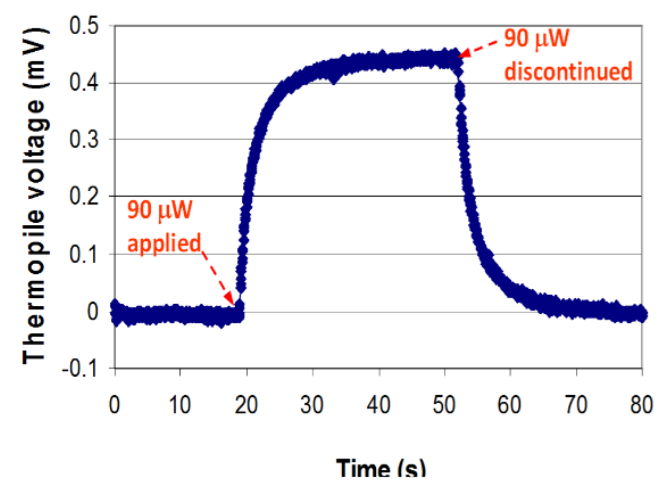

(c)

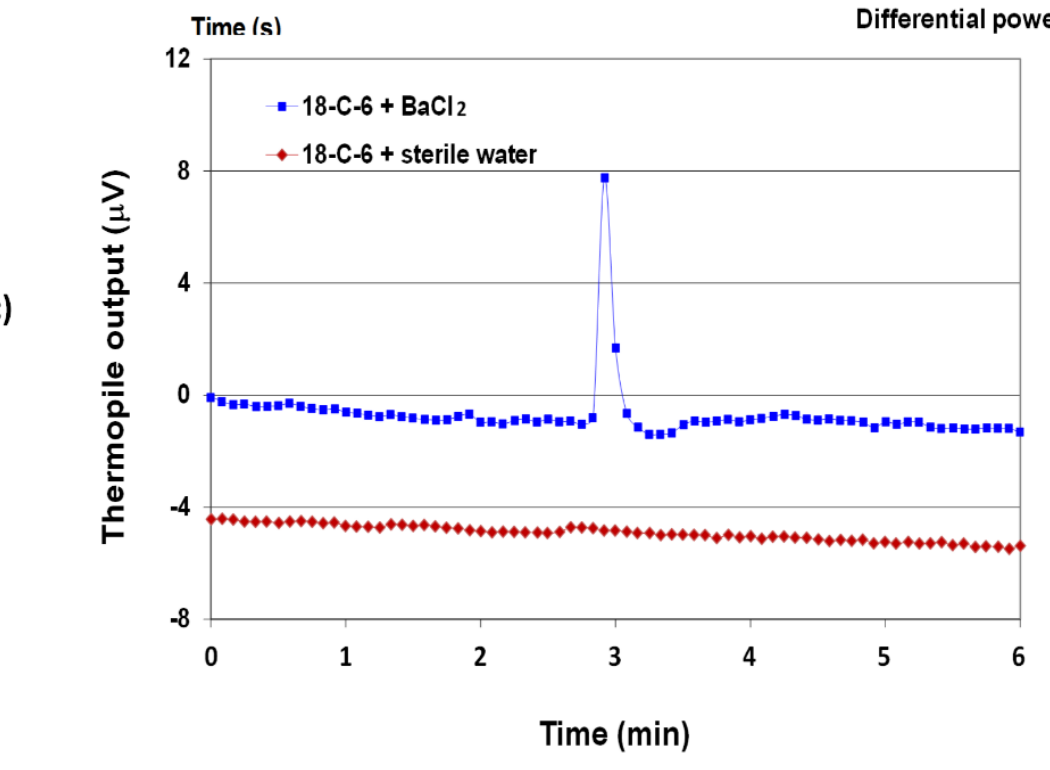

(b)

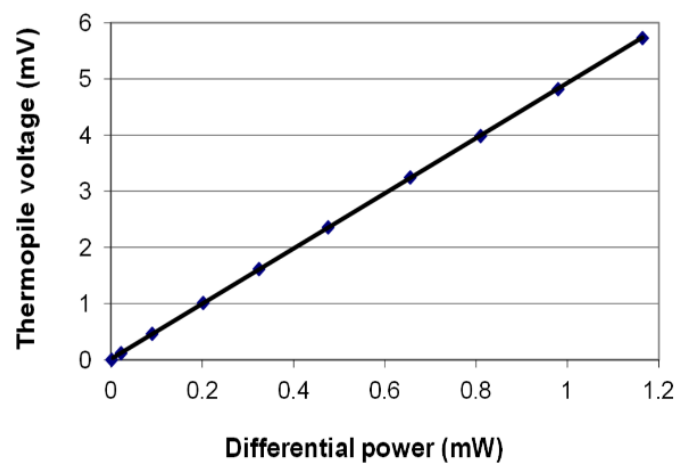

Figure 4. Response of the ITC microdevice to on-chip differential heating between the calorimetric chambers. (a) Transient responses to application and removal of a constant differential power; (b) steady-state response to different values of differential power; (c) timeresolved device output upon introduction of $5 \mathrm{mM} 18-\mathrm{C}-6$ and $4 \mathrm{mM} \mathrm{BaCl} 2$ (each $0.5 \mu \mathrm{L}$ ), compared with measurement of $5 \mathrm{mM} 18-\mathrm{C}-6$ and sterile water. (The traces are plotted with an intentionally added offset of $-4 \mu \mathrm{V}$ for clarity.) 
Using the standard chemical reaction of $18-\mathrm{C}-6$ and $\mathrm{BaCl}_{2}$ in sterile water, we verified the stability of the baseline in the device output. In the measurement, $0.5 \mu \mathrm{L}$ each of $\mathrm{BaCl}_{2}(4 \mathrm{mM})$ solution and sterile water were introduced into the reaction and reference chambers simultaneously within $1 \mathrm{~s}$. The measured device's thermopile output varied only moderately with time (Figure 4(c)), showing no appreciable thermal activity from dilution of 18-C-6 (which commonly exists in conventional ITC instruments [36]) and providing a stable baseline. We then examined the time-resolved thermopile voltage upon simultaneous introduction of $0.5 \mu \mathrm{L}$ each of 18-C-6 $(5 \mathrm{mM})$ and $\mathrm{BaCl}_{2}(4 \mathrm{mM})$ into the reaction chamber (flow rate: $\left.50 \mu \mathrm{L} / \mathrm{min}\right)$, and $0.5 \mu \mathrm{L}$ each of $18-\mathrm{C}-6(5 \mathrm{mM})$ and sterile water into the reference chamber (flow rate: $50 \mu \mathrm{L} / \mathrm{min}$ ) (Figure 4(c)). The device output exhibited a reaction-specific spike attributable to the exothermic nature of the binding between 18-C-6 and $\mathrm{BaCl}_{2}$. The spike was determined to appear within $1 \mathrm{~s}$ of introduction of the reagents; this minimal delay reflected the rapid thermal response of the microdevice as described above. In addition, the spike spanned approximately $20 \mathrm{~s}$ corresponding to the duration of heat evolved from the reaction. We estimated the time for the ligand and receptor solutions to become fully mixed as they entered the reaction chamber by numerical simulation of convective-diffusive mass transfer. This mixing time was approximately $0.2 \mathrm{~s}$ and was negligibly small compared with the spike duration, which was hence determined to be determined primarily by the reaction kinetics.

\subsection{Verification of ITC Measurements}

We first used the model reaction system of $18-\mathrm{C}-6$ and $\mathrm{BaCl}_{2}$ to verify that our microdevice can appropriately function as an ITC instrument [37]. Rather than measuring the heat evolved with the addition of several aliquots of $\mathrm{BaCl}_{2}$ to $18-\mathrm{C}-6$ as in conventional instruments [38], microdevice-based ITC was performed in a series of measurements [28], with the temperature of 
the support stage for the microdevice maintained at $23{ }^{\circ} \mathrm{C}$ or $35^{\circ} \mathrm{C}$. Each measurement used a fixed concentration of 18-C-6 (5 mM) and one of a set of concentrations (in the range of 0.5-10 $\mathrm{mM}$ ) of $\mathrm{BaCl}_{2}$, allowing the molar ratio of $\mathrm{BaCl}_{2}$ to $18-\mathrm{C}-6$ to be varied from 0.1 to 2 . Measurement at each molar ratio occurred in a duration of approximately $3 \mathrm{~min}$, with the entire ITC measurement completed within approximately $21 \mathrm{~min}$.

At each of the controlled temperatures 23 and $35^{\circ} \mathrm{C}$, the baseline-subtracted thermopile voltage from the device (Figure 5(a) \& (b)) exhibited spikes consistent with the titration reactions. At both temperatures, a spike in the device output was observed in response to each titration, with a magnitude increasing consistently with the titration number. This reflects that an increasingly larger amount of $18-\mathrm{C}-6$ participated in the reaction as more $\mathrm{BaCl} 2$ molecules were introduced. At all of the molar ratios, no significant baseline drift after the completion of each binding, this holds potential to offer highly stable ITC measurements on this microdevice-based setup. 
(a)

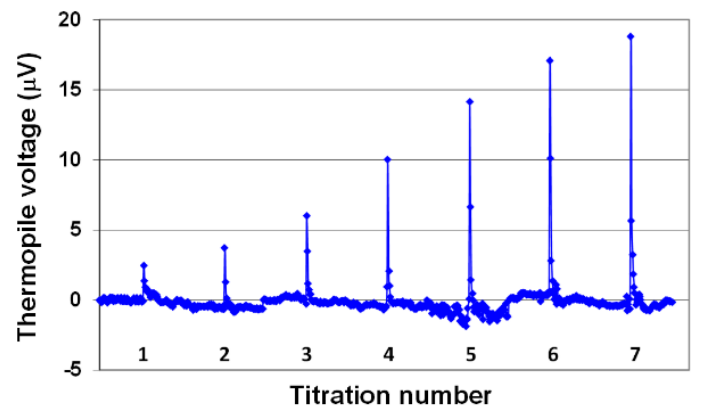

(b)

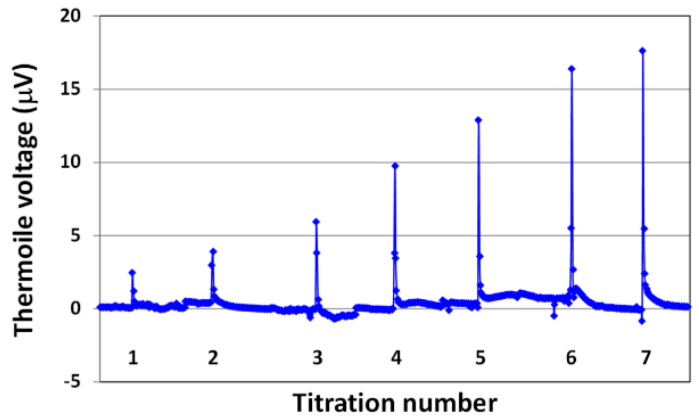

(c)

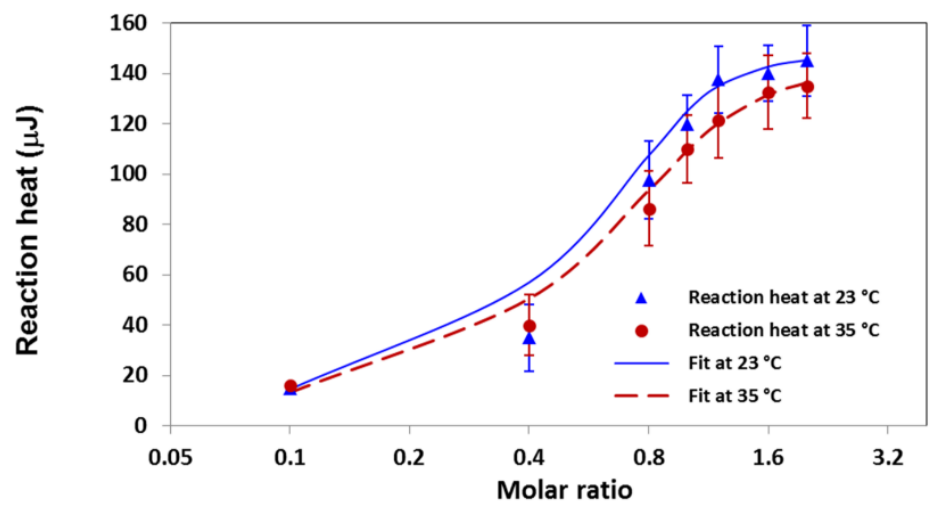

Figure 5. Baseline-subtracted device output from the binding of $18-\mathrm{C}-6(5 \mathrm{mM})$ with $\mathrm{BaCl}_{2}$ in seven titrations. Titration numbers corresponded to molar ratios of $\mathrm{BaCl}_{2}$ to $18-\mathrm{C}-6$ of $0.1,0.4$, $0.8,1.0,1.2,1.6$ and 2.0, respectively. (a) Measurements at $23{ }^{\circ} \mathrm{C}$; (b) measurements at $35{ }^{\circ} \mathrm{C}$; (c) reaction heat as a function of the molar ratio of $\mathrm{BaCl}_{2}$ to $18-\mathrm{C}-6$ based on triplicate measurements at a controlled temperature of 23 or $35^{\circ} \mathrm{C}$.

The molar ratio-dependent thermopile voltage at each controlled temperature was then used to calculate the differential thermal power according to Eq. (3). The reaction heat was calculated by integrating the thermal power over the entire time period of reaction. A binding isotherm was then obtained depicting the reaction heat as a function of the molar ratio $[28,38]$, and used to determine the thermodynamic properties of the binding system by least squares fitting to Eq. (2). The binding isotherm of the reactions of $18-\mathrm{C}-6$ and $\mathrm{BaCl}_{2}$, as well as the best fits, is shown in 
Figure 5(c), with error bars representing the standard deviation calculated from triplicates of measurements at each molar ratio. It can be seen that the reaction heat increased monotonically with the molar ratio (i.e., as an increasing amount of $\mathrm{BaCl}_{2}$ was added to 18-C-6), albeit at a decreasing rate until approaching a saturation value, reflecting that with the addition of $\mathrm{BaCl}_{2}$, an increasingly diminished amount of 18-C-6 was available for reaction.

From the binding isotherms at the controlled temperatures, it can be seen that the stoichiometry remained approximately $N=1$ at both temperatures, corresponding to the monovalent binding of $18-\mathrm{C}-6$ and $\mathrm{BaCl}_{2}$. On the other hand, the equilibrium binding constant $K_{\mathrm{B}}$ was determined to be $6.0 \times 10^{3} \mathrm{M}^{-1}$ and $2.8 \times 10^{3} \mathrm{M}^{-1}$ at $23{ }^{\circ} \mathrm{C}$ and $35{ }^{\circ} \mathrm{C}$, respectively. The lower $K_{\mathrm{B}}$ at $35{ }^{\circ} \mathrm{C}$ indicated weaker binding at the higher temperature, reflecting the increased tendency for the reaction product to dissociate [36]. In addition, the molar enthalpy change $\Delta H$ was determined to be 30.0 and $27.8 \mathrm{~kJ} / \mathrm{mol}$ at $23{ }^{\circ} \mathrm{C}$ and $35^{\circ} \mathrm{C}$, respectively. These properties are consistent with published data using conventional instruments [36]. We noticed that in the microdevice-based ITC experiments, the binding affinity decreased more significantly with temperature when compared to measurements made with commercial ITC instruments. This could be attributed to the increased loss of reaction heat within the mixing region at the elevated temperature, which could be addressed by an improved micromixer design that minimizes the time prior to the entry of the reactants into the reaction chamber.

\subsection{Characterization of Biomolecular Interaction}

We then applied the ITC microdevice to characterization of biomolecular interactions, using RNase A and 2'CMP as a representative ligand-protein binding system at different controlled 
temperatures of $23^{\circ} \mathrm{C}$ and $35^{\circ} \mathrm{C}$. The small molecule $2^{\prime} \mathrm{CMP}$ is known to be a strong inhibitor of substrates that bind to the active site of RNase A [38]. Both reagents were prepared in $50 \mathrm{mM}$ potassium acetate buffer, $\mathrm{pH}$ 5.5. The concentration of RNase A was fixed at a practically relevant level of $2 \mathrm{mM}$ [39] (hence with mass consumption lower than $10 \mu \mathrm{g}$ per molar ratio) while the $2^{\prime} \mathrm{CMP}$ was prepared at a series of concentrations in the range of $0.2-4 \mathrm{mM}$ to allow for the 2'CMP-RNase A molar ratio to be varied between 0.1 and 2 .

At each controlled temperature, the device's thermopile output (subtracted with the baseline, which was obtained with the reaction and reference chambers both filled with $0.5 \mu \mathrm{L}$ each of 2 $\mathrm{mM}$ RNase A and $50 \mathrm{mM}$ potassium acetate buffer) exhibited clearly visible spikes (subtracted with the baseline, which was obtained with the reaction and reference chambers both filled with $0.5 \mu \mathrm{L}$ each of $2 \mathrm{mM}$ RNase A and $50 \mathrm{mM}$ potassium acetate buffer) in response to the titrations of 2'CMP into RNase A (Figure 6(a) \& (b)). Also, it was observed that the spike spanned times for the binding of RNase A and 2' CMP at different molar ratios were approximately $20 \mathrm{~s}$ as well, so that each measurement was completed within 3-4 min which included time required for reagent introducing and device stabilization, thus leading to a typical duration of 20-30 min for a complete ITC run. Compared with those on conventional ITC instruments that typically require a total analysis time of 1-2 hours [36], our approach allows for considerable reduction in analysis time.

As the molar ratio of $2^{\prime} \mathrm{CMP}$ to RNase A increased from 0.1 to 2 , the spike magnitude increased consistently and eventually became saturated. This indicated that as the amount of 2'CMP increases, a larger amount of RNase A participates in the binding until the maximum possible amount of RNase A-2'CMP complex was formed. The measured molar ratio-dependent thermopile voltage at each controlled temperature was again used to compute the differential 
thermal power (Eq. (3)), which was then integrated to obtain the reaction heat, thereby obtaining a binding isotherm as shown in Figure 6(c) (error bars again represent standard deviations from triplicate measurements). The reaction heat increased with the molar ratio as a result of the increasing amount of 2'CMP presented to RNase A, and gradually approached a saturation value, reflecting again that a diminished amount of RNase A molecules was available to react with the added 2'CMP.

Also as shown in the figure, the binding isotherm at each temperature was used to determine the thermodynamic properties of the binding system by least squares fitting to Eq. (2). The stoichiometry was approximately $N=1$ at both of the controlled temperatures, indicating monovalent binding between RNase A and 2'CMP. The equilibrium binding constant $K_{\mathrm{B}}$ was determined to be $9.0 \times 10^{4}$ and $4.0 \times 10^{4} \mathrm{M}^{-1}$ at $23{ }^{\circ} \mathrm{C}$ and $35^{\circ} \mathrm{C}$, respectively. This again showed weaker binding affinity at elevated temperatures. In addition, the total molar enthalpy change $\Delta H$ was determined to be $-52.3 \mathrm{~kJ} / \mathrm{mol}$ at $23{ }^{\circ} \mathrm{C}$ and $-56.0 \mathrm{~kJ} / \mathrm{mol}$ at $35{ }^{\circ} \mathrm{C}$, respectively. The larger absolute change in molar enthalpy at $35^{\circ} \mathrm{C}$ suggests a decrease in heat capacity due to a decrease in exposure of hydrophobic groups to water when 2'CMP binds to RNase [34]. These results are consistent with published data for the 2'CMP and RNase A binding system using conventional instruments [38], corroborating the correctness of our microdevice-based ITC measurements. 
(a)

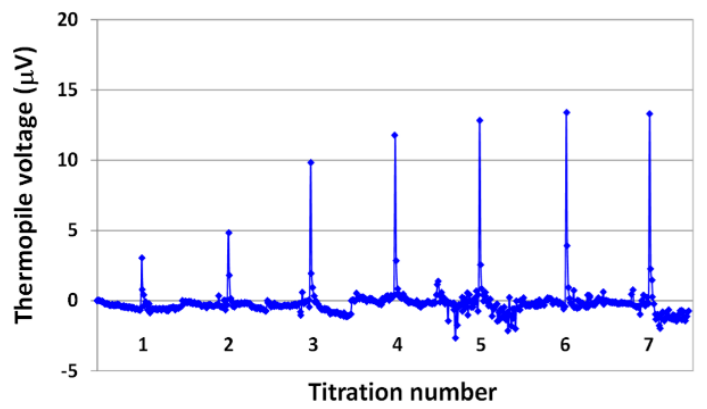

(b)



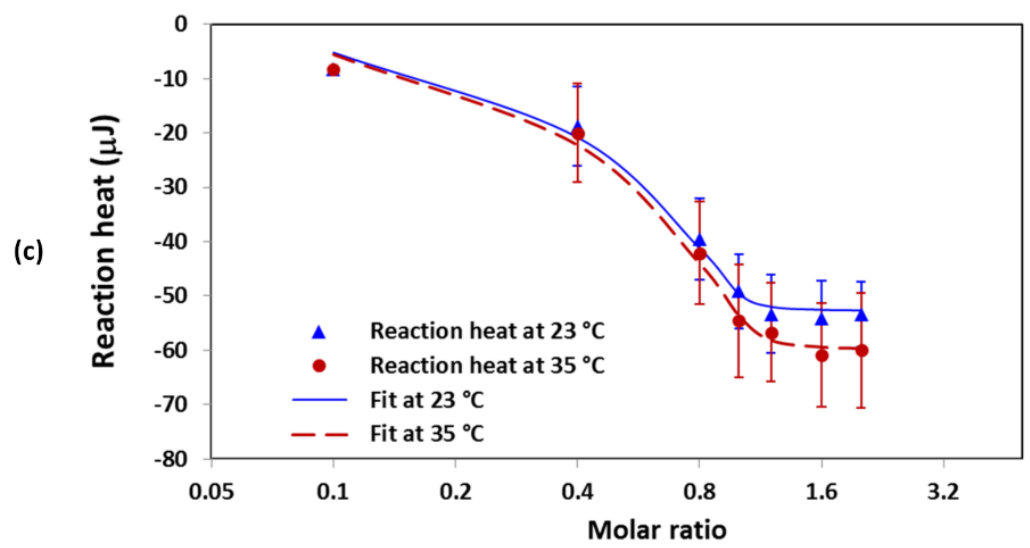

Figure 6. Baseline-subtracted device output from the binding of RNase A (2 mM) with 2'CMP in seven titrations. Titration numbers corresponded to molar ratios of 2' $\mathrm{CMP}$ to RNase A of 0.1, $0.4,0.8,1.0,1.2,1.6$ and 2.0 , respectively. (a) Measurements at $23{ }^{\circ} \mathrm{C}$; (b) measurements at $35^{\circ} \mathrm{C}$; (c) reaction heat as a function of the molar ratio of $2^{\prime} \mathrm{CMP}$ to RNase A based on triplicate measurements at a controlled temperature of 23 or $35^{\circ} \mathrm{C}$.

\section{CONCLUSIONS}

This paper presents a microfabrication-based approach to integrated, quantitative ITC characterization of biomolecular interactions. The approach integrates microfabricated differential calorimetric sensors with microfluidic titration, in which microliter-level reactants are mixed at a series of molar ratios in a constant volume and at a controlled temperature, while 
the reaction heat is measured. A microdevice is used to implement the approach and consists of thermally isolated microchambers with minimized liquid evaporation to provide a constantvolume reaction environment, passive micromixers for titration of reactants, and a thermoelectric sensor for differential detection of the reaction heat. Quantitative ITC measurements can thus be performed at user-specified temperature set points, within considerably reduced analysis times, without dilution-induced baseline shifts that often affect conventional ITC instruments, and potentially at low cost.

This ITC approach has been demonstrated with a microdevice that consists of a microfluidic structure bonded to a thermal control and sensing substrate. The PDMS microfluidic structure includes two calorimetric chambers each thermally isolated by an underlying freestanding polyimide diaphragm and surrounding air cavities, and connected to a passive chaotic micromixer. On the thermal substrate, the freestanding diaphragms are integrated with a thinfilm Sb-Bi thermopile for differential measurement of thermal power in the chambers. The diaphragms are also each integrated with a thin-film resistive micro temperature sensor and a microheater for in-situ temperature monitoring and on-chip device calibration. During operation, the microdevice temperature is controlled at a desired setpoint. Ligand and receptor solutions are introduced at a given molar ratio, mixed in the appropriate micromixer and delivered into the reaction chamber. Meanwhile, ligand-free buffer and receptor solutions are introduced, mixed and delivered into the reference chamber. The differential thermal power in the chambers is determined from the baseline-corrected thermopile voltage. Data from these measurements at a series of ligand-receptor molar ratios are used to compute the binding parameters such as the stoichiometry, enthalpy of the binding, and equilibrium binding. 
We have demonstrated ITC measurements of the binding of $18-\mathrm{C}-6$ with $\mathrm{BaCl} 2$, and the binding of RNase A with 2'CMP, completing quantitative ITC measurements at low cost, under each of the prespecified isothermal conditions, with considerably reduced analysis times. The resulting data are then used to compute the thermodynamic profile of the biomolecular systems. With the temperature controlled at 23 and $35{ }^{\circ} \mathrm{C}$, respectively, the thermodynamic properties of the biomolecular interaction, including the stoichiometry, equilibrium binding constant, and enthalpy change, were obtained and found to agree with published results from commercially available instruments. For the protein-ligand binding system of RNase A and 2'CMP, quantitative ITC measurements were performed with a reaction volume of approximately $0.7 \mu \mathrm{L}$ at concentrations as low as $2 \mathrm{mM}$, which is practically relevant and can be further improved by improved device design and operation (e.g., enhanced thermal isolation of calorimetric chambers, reduced heat loss during mixing, and optimized operating parameters). The thermodynamic

properties of the RNase A and 2'CMP binding were again obtained at the controlled temperatures of 23 and $35^{\circ} \mathrm{C}$, respectively, and found in agreement with published data. These results demonstrate the potential of our approach for efficient quantitative ITC characterization of biomolecular interactions in biomedical applications.

\section{ACKNOWLEDGMENTS}

This research has been supported by the National Science Foundation (Award Nos. DBI0650020 and CBET-0854030). We are also grateful to Prof. Jonathan B. Chaires for providing 2'CMP and RNase A samples used in our testing and Xiangsong Feng, Yibo Zhu for their assistance in the simulation and data fitting. 


\section{REFERENCES}

[1] S. Leavitt and E. Freire, "Direct measurement of protein binding energetics by isothermal titration calorimetry," Current Opinion in Structural Biology, vol. 11, pp. 560-566, Oct 2001.

[2] U. v. Ah, D. Wirz, and A. Daniels, "Isothermal micro calorimetry - a new method for MIC determination: results for 12 antibotics and reference strains of E. coli and S. aureus," BMC Microbiology, vol. 9, p. 106, 2009.

[3] A. L. Feig, "Review: applications of isothermal titration calorimetry in RNA biochemistry and biophysics," Biopolymers, vol. 87, pp. 293-301, 2007.

[4] T. G. A. Lonhienne and D. J. Winzor, "A potential role for isothermal calorimetry in studies of the effects of thermodynamic non-ideality in enzyme-catalyzed reactions," Journal of Molecular Recognition, vol. 17, pp. 351-361, 2004.

[5] J. E. Ladbury, "Isothermal titration calorimetry: application to structure-based drug design," Thermochimica Acta, vol. 380, pp. 209-215, 2001.

[6] M. A. A. O'Neill and S. Gaisford, "Applications and use of isothermal calorimetry in pharmaceutical development," International Journal of Pharmaceutics, vol. 417, pp. 83-93, 2011.

[7] L. S. Roselin, M.-S. Lin, P.-H. Lin, Y. Chang, and W.-Y. Chen, "Recent trends and some applications of isothermal titration calorimetry in biotechnology," Biotechnology Journal, vol. 5, pp. 85-98, 2010.

[8] J. B. Chaires, "Calorimetry and thermodynamics in drug design," Annual Review of Biophysics, vol. 37, pp. 135-151, 2008.

[9] A. W. v. Herwaarden, "Overview of calorimeter chips for various applications," Thermochimica Acta, vol. 432, pp. 192-201, 2005.

[10] R. E. Cavicchi, G. E. Poirier, N. H. Tea, M. Afridi, D. Berning, A. Hefner, et al., "Micro-differential scanning calorimeter for combustible gas sensing," Sensors and Actuators B: Chemical, vol. 97, pp. 22-30, 2004.

[11] D. W. Cooke, K. J. Michel, and F. Hellman, "Thermodynamic measurements of submilligram bulk samples using a membrane-based "calorimeter on a chip"," Review of Scientific Instruments, vol. 79, p. 053902, 2008.

[12] E. lervolino, A. W. v. Herwaarden, and P. M. Sarro, "Calorimeter chip calibration for thermal characterization of liquid samples," Thermochimica Acta, vol. 492, pp. 95-100, 2009.

[13] W. Lee, W. Fon, B. W. Axelrod, and M. L. Roukes, "High-sensitivity microfluidic calorimeters for biological and chemcial applications," PNAS, vol. 106, pp. 15225-15230, 2009.

[14] Y. Zhang and S. Tadigadapa, "Calorimetric biosensors with integrated microfluidic channels," Biosnesors and Bioelectronics, vol. 19, pp. 1733-1743, 2004.

[15] Y. Zhang and S. Tadigadapa, "Thermal characterization of liquids and polymer thin films using a microcalorimeter," Applied Physics Letters, vol. 86, p. 034101, 2005.

[16] C. Pradere, C. Hany, J. toutain, and J.-C. Batsale, "Thermal analysis for velocity, kinetics, and enthalpy reaction measurements in microfluidic devices," Experimental Heat Transfer, vol. 23, pp. 44-62, 2010.

[17] N. Inomata, M. Toda, M. Sato, A. Ishijima, and T. Ono, "Pico calorimeter for detection of heat produced in an individual brown fat cell," Applied Physics Letters, vol. 100, p. 154104, 2012.

[18] G. V. Casquillas, F. Bertholle, M. L. Berre, S. Meance, L. Malaquin, J. J. Greffet, et al., "Thermresistance based micro-calorimeter for continuous chemical enthalpy measurements," Microelectronic Engineering, vol. 85, pp. 1367-1369, 2008. 
[19] V. Baier, R. Fodisch, A. Ihring, E. Kessler, J. Lerchner, G. Wolf, et al., "Highly sensitive thermopile heat power sensor for micro-fluid calorimetry of biochemical processes," Sensors and Actuators a-Physical, vol. 123-24, pp. 354-359, Sep 232005.

[20] A. J. Taberner, I. W. Hunter, R. S. Kirton, P. M. F. Nielsen, and D. S. Loiselle, "Characterization of a flow-through microcalorimeter for measuring the heat production of cardiac trabeculae," Review of Scientific Instruments, vol. 76, Oct 2005.

[21] F. E. Torres, P. Kuhnt, D. De Bruyker, A. G. Bell, M. V. Wolkin, E. Peeters, et al., "Enthalpy arrays," Proceedings of the National Academy of Sciences of the United States of America, vol. 101, pp. 9517-9522, Jun 292004.

[22] B. S. Kwak, B. S. Kim, H. H. Cho, J. S. Park, and H. I. Jung, "Dual thermopile intergated microfluidic calorimeter for biochemical thermodynamics," Microfluidics and Nanofluidics, vol. 5, pp. 255262, 2008.

[23] B. Wang and Q. Lin, "A MEMS Differential-Scanning-Calorimetric Sensor for Thermodynamic Characterization of Biomolecules," Journal of Microelectromechanical Systems, vol. 21, pp. 1165-1171, Oct 2012.

[24] Y. Jia, B. Wang, Z. Zhang, and Q. Lin, "A polymer-based MEMS differential scanning calorimeter," Sensors and Actuators A: Physical, Article In Press.

[25] C. Hany, H. Lebrun, C. Pradere, J. Toutain, and J.-C. Batsale, "thermal analysis of chemical reaction with a continuous microfluidic calorimeter," Chemical Engineering Journal, vol. 160, pp. 814-822, 2010.

[26] B. Lubbers and F. Baudenbacher, "Isothermal titration calorimetry in nanoliter droplets with subsecond time constants," Analytical Chemistry, vol. 83, pp. 7955-7961, 2011.

[27] J. Xu, R. Reiserer, J. Tellinghuisen, J. P. Wikswo, and F. J. Baudenbacher, "A mcirofabricated nanocalorimeter: design, characterization, and chemical calibration," Analytical Chemistry, vol. 80, pp. 2728-2733, 2008.

[28] M. I. Recht, D. D. Bruyker, A. G. bell, M. V. Wolkin, E. Peeters, G. B. Anderson, et al., "Enthalpy array analysis of enzymatic and binding reactions," Analytical Biochemistry, vol. 377, pp. 33-39, 2008.

[29] C. Sanders, "Biomolecular Ligand-Receptor Binding Studies: Theory, Practice, and Analysis," http://structbio.vanderbilt.edu/sanders/Binding Principles 2010.pdf, 2010.

[30] Malvern, "Isothermal Titration Calorimetry," http://www.malvern.com.

[31] L. MicroCal, "ITC data analysis in Origin: Tutorial Guide," 2004.

[32] L. Wang, B. Wang, and Q. Lin, "Demonstration of MEMS-based differential scannign calorimetry for determining thermodynamic properties of biomolecules," Sensors and Actuators B: Chemical, vol. 134, pp. 953-958, 2008.

[33] A. D. Stroock, S. K. W. Dertinger, A. Ajdari, I. Mezic, H. A. Stone, and G. M. Whitesides, "Chaotic mixer for microchannels," Science, vol. 295, pp. 647-651, 2002.

[34] S. Milev, "Isothermal titration calorimetry: Principles and experimental design," 2013.

[35] M. Duff, J. Grubbs, and E. Howell, "Isothermal Titration Calorimetry for Measuring Macromolecule-Ligand Affinity," Journal of Visualized Experiments, 2011.

[36] MicroCal. (2004). Use of isothermal titration calorimetry to measure enzyme kinetics parameters [Application note]. Available: www.microcalorimetry.com

[37] J. Tellinghuisen, "Designing isothermal titration calorimetry experiments for the study of 1:1 binding: problem with the "standard protocol"," Analytical Biochemistry, vol. 424, pp. 211-220, 2012.

[38] T. Wiseman, S. Williston, J. F. Brandts, and L.-N. Lin, "Rapid measurements of binding constants and heats of binding using a new titration calorimeter," Analytical Biochemistry, vol. 179, pp. 131-137, 1989. 
[39] N. Demarse and C. Quinn, "Determination of a protein ligand interaction via continuous isothermal titration calorimetry," www.tainstruments.com, 2011. 